\title{
Cahiers franco-canadiens de l'Ouest
}

\section{Écrire pour l'Autre : les publications de langue anglaise de deux sociétés historiques en milieu minoritaire}

\section{Stéphanie St-Pierre}

Volume 29, numéro 1, 2017

Langue, culture et histoire d'une Amérique francophone plurielle

URI : https://id.erudit.org/iderudit/1041199ar

DOI : https://doi.org/10.7202/1041199ar

Aller au sommaire du numéro

\section{Éditeur(s)}

Presses universitaires de Saint-Boniface (PUSB)

ISSN

0843-9559 (imprimé)

1916-7792 (numérique)

Découvrir la revue

Citer cet article

St-Pierre, S. (2017). Écrire pour l'Autre : les publications de langue anglaise de deux sociétés historiques en milieu minoritaire. Cahiers franco-canadiens de l'Ouest, 29(1), 83-114. https://doi.org/10.7202/1041199ar

\section{Résumé de l'article}

Les sociétés historiques de langue française jouent un rôle important dans la prise de conscience historiographique des Canadiens français de la seconde moitié $\mathrm{du} \mathrm{XIX}{ }^{\mathrm{e}}$ siècle au tournant du XX⿳亠丷厂犬 siècle. La Société historique franco-américaine (SHFA) et la Société historique de Saint-Boniface (SHSB) ne font pas exception. Puisqu'elles développent un discours sur le passé, elles contribuent à façonner le territoire historique dans l'historiographie et à élaborer un discours d'enracinement pour les populations de langue française qui habitent les régions qu'elles desservent. Largement influencées par leur situation en milieu minoritaire, les deux sociétés historiques à l'étude sont ancrées dans leur région de par les thématiques abordées et le discours qui se rapportent à leur fondation. Si elles cherchent principalement à jouer le rôle de vecteur d'une histoire par et pour la population de langue française, elles choisissent toutes deux de publier des textes historiques en anglais, et ce, peu de temps après leur fondation. Par une analyse de ces deux publications de langue anglaise, cet article permettra de mieux saisir la place qu'occupe la représentation du territoire historique et du discours d'enracinement dans l'étude de l'histoire de soi pour l'Autre, qui est majoritaire et anglophone.
Tous droits réservés (C Centre d'études franco-canadiennes de l'Ouest (CEFCO) et Presses universitaires de Saint-Boniface (PUSB), 2017
Ce document est protégé par la loi sur le droit d'auteur. L'utilisation des services d'Érudit (y compris la reproduction) est assujettie à sa politique d'utilisation que vous pouvez consulter en ligne.

https://apropos.erudit.org/fr/usagers/politique-dutilisation/ 


\title{
Écrire pour l'Autre: les publications de langue anglaise de deux sociétés historiques en milieu minoritaire ${ }^{1}$
}

\author{
Stéphanie St-Pierre \\ Université de Montréal
}

\begin{abstract}
RÉSUMÉ
Les sociétés historiques de langue française jouent un rôle important dans la prise de conscience historiographique des Canadiens français de la seconde moitié du XIX siècle au tournant du $X^{\mathrm{e}}$ siècle. La Société historique franco-américaine (SHFA) et la Société historique de Saint-Boniface (SHSB) ne font pas exception. Puisqu'elles développent un discours sur le passé, elles contribuent à façonner le territoire historique dans l'historiographie et à élaborer un discours d'enracinement pour les populations de langue française qui habitent les régions qu'elles desservent. Largement influencées par leur situation en milieu minoritaire, les deux sociétés historiques à l'étude sont ancrées dans leur région de par les thématiques abordées et le discours qui se rapportent à leur fondation. Si elles cherchent principalement à jouer le rôle de vecteur d'une histoire par et pour la population de langue française, elles choisissent toutes deux de publier des textes historiques en anglais, et ce, peu de temps après leur fondation. Par une analyse de ces deux publications de langue anglaise, cet article permettra de mieux saisir la place qu'occupe la représentation du territoire historique et du discours d'enracinement dans l'étude de l'histoire de soi pour l'Autre, qui est majoritaire et anglophone.
\end{abstract}

Mots clés : Sociétés historiques, discours historique, identité, territoire, Canada français, Franco-Américains, Franco-Manitobains. 


\begin{abstract}
French language historical societies, such as the Société historique franco-américaine (SFHA) and the Société historique de Saint-Boniface (SHSB) play a key role in the development of a historic consciousness in Manitoba and New England. Their mere existence in a minority setting helps establish the boundaries of the territory upon which the historical accounts of the French and FrenchCanadians are set. Although their publications were generally geared towards a French Canadian readership, both historical societies published texts in English, destined for the majority with whom they were destined to cohabitate. How has writing for "others" influenced their interpretation of historic events? And how has the underlying identity discourse been presented to the English majority? By conducting a comparative analysis of two English language publications, as well as a selection of French language texts and publications, this article wishes to better understand the impact of language and readership on the elaboration of an identity discourse, firmly rooted in the past.
\end{abstract}

Key words: Historical society, historic discourse, identity, territory, French Canada, Franco-Americans, FrancoManitobans.

Si les grandes migrations canadiennes-françaises ${ }^{2}$ et les communautés de langue française qu'elles sèment partout sur le territoire attirent l'attention des chercheurs ${ }^{3}$, les retombées historiographiques de ce mouvement expansionniste sont cependant moins étudiées. Pourtant, ce mouvement migratoire à partir du Québec crée des contextes favorables à la formulation de discours sur le passé sur lesquels on devrait se pencher, afin de mieux comprendre le rôle de ces derniers dans la construction identitaire des populations vivant en milieu minoritaire et la mise en récit de l'histoire.

Les sociétés historiques constituent le vecteur par excellence du discours d'enracinement, soit du discours identitaire portant sur le territoire historique. Leurs publications sont signées par des amateurs de l'histoire - clercs et laïques issus des professions libérales auxquels se grefferont, plus 
tard, certains historiens professionnels - ce qui enrichit la complexité de ce discours. De par leur intérêt pour le territoire habité qu'évoque le mandat régional, ces sociétés historiques accordent une place importante à l'histoire de la région, au « fait français ", à l'origine française (ou canadienne-française) et à l'appartenance à la nation.

Le discours d'enracinement qui est formulé par les sociétés historiques s'observe dès leur fondation puisque l'acte même de fonder ces sociétés sert de gage ou d'élément de mesure dans l'élaboration d'un territoire historique. Ces sociétés " pensent » le territoire en s'appuyant souvent sur des moments clés de l'histoire des populations de langue française dans ces espaces. Elles sont fondées dans le but de faire connaître l'histoire des régions où l'écriture d'une histoire est souvent inexistante ou lacunaire. Elles sont établies parce que les fondateurs croient qu'il y a une histoire à raconter. Dans cette optique, les sociétés historiques régionales agissent comme des institutions complémentaires à l'historiographie at large et le rapport qu' elles entretiennent avec l'Autre est d'autant plus digne d'intérêt qu'il s'inscrit dans un rapport à soi en pleine évolution.

C'est ce rapport à l'Autre qui sera étudié dans le cadre de cet article, par l'analyse de deux publications de langue anglaise produites par deux sociétés historiques de langue française, soit la Société historique franco-américaine (SHFA), fondée en 1899, et la Société historique de Saint-Boniface (SHSB), établie en 1902. Ces deux sociétés sont fondées dans ce qui constitue une première vague de sociétés historiques de langue française en Amérique du Nord (St-Pierre, 2015). Elles suivent les traces de la Société historique de Montréal, la première société historique homogène de langue française, fondée en 1858.

Au cours de la décennie suivant leur création, ces deux sociétés historiques sont très actives de par leurs conférences et leurs publications. Si les activités de ces sociétés se déroulent généralement en français, on compte néanmoins quelques publications de langue anglaise. Bien que peu nombreuses, ces publications offrent une vitrine intéressante sur la représentation de soi pour l'Autre. Qui plus est, ces publications de langue anglaise paraissent assez rapidement après la fondation des deux sociétés savantes, soit en 1909 du côté de la SHFA (Favreau et al., 1909) et en 1916 du côté de la SHSB (Prud'homme, 1916). 
Il s'agit là d'un indice selon lequel la publication dans la langue de la majorité revêt une certaine importance pour les dirigeants des deux sociétés. De plus, les deux textes de langue anglaise à l'étude sont rédigés par des membres fondateurs des deux sociétés historiques.

En raison du contexte minoritaire dans lequel œuvrent ces deux sociétés historiques, les publications de langue anglaise nous permettent de nous interroger sur le rôle de l'Autre à la fois comme interlocuteur et comme public cible. Cet Autre dont il est question est le majoritaire anglophone avec qui le groupe minoritaire francophone est amené à dialoguer, tant dans l'univers du quotidien - par sa réalité démographique - que dans le monde des idées, où l'appartenance, les droits et les revendications se côtoient.

Les membres fondateurs des deux sociétés historiques participent à l'élaboration du discours d'enracinement pour soi, par la fondation même de la société. Ce sont les fondateurs qui élaborent les lignes directrices des sociétés par le mandat ou la charte, et qui se sentent investis de la responsabilité de mettre en lumière l'histoire de la région pour les autres Canadiens français qui l'habitent. Qui plus est, ils figurent aussi parmi les auteurs des textes en anglais qui sont à l'étude dans le présent article. Étant donné ce double rôle des fondateurs dans l'élaboration d'un discours d'enracinement pour soi et pour l'Autre, la présente analyse juxtapose le discours par rapport à la fondation aux représentations historiques repérées dans les deux textes de langue anglaise. Dans un premier temps, cet article explore le contexte de fondation des deux sociétés historiques et la représentation du passé et du territoire historique qui en découle dans deux régions de la diaspora canadienne-française : l'Ouest canadien et la Nouvelle-Angleterre. Dans un deuxième temps, cet article s'interroge sur le discours d'enracinement véhiculé par les publications de langue anglaise de ces deux sociétés. Comment présente-t-on le territoire historique, l'appartenance et les particularités de la "race» dans un texte rédigé pour l'Autre? Le discours d'enracinement comporte-t-il des traces d'hybridité identitaire ou de compromis, influencé par ce désir d'être lu et, qui plus est, compris par le groupe cible? 


\section{Représentations historiques et discours d'enracinement}

Le discours d'enracinement et la représentation du territoire historique dans les historiographies de langue française en milieu minoritaire servent de point $d^{\prime}$ ancrage de notre analyse. S'ajoutent à ces deux concepts celui d'acte fondateur ou d'ouvrage pionnier. Le concept de discours d'enracinement correspond à une forme de discours fortement empreinte de références au passé, et plus particulièrement à l'histoire vécue dans un lieu ou un territoire donné, par le groupe d'appartenance. Ce discours d'enracinement sert ainsi à ancrer ou à enraciner ce groupe d'appartenance à un lieu donné ou, par ricochet, à légitimer la présence par l'enracinement dans le territoire. La référence au territoire et au rôle que ce territoire joue dans l'histoire - le territoire historique - constitue pour sa part l'expression la plus concrète du discours d'enracinement. Les références à ce territoire historique $\mathrm{s}^{\prime}$ inscrivent dans la trame narrative de l'histoire du Canada français et de l'Amérique par le biais d'événements marquants qui servent souvent à démontrer l'ancienneté sur le territoire: les explorations à l'époque de la Nouvelle-France, la colonisation, la fondation de paroisses, etc.

Cette démarche s'appuie donc sur le principe, déjà proposé par certains chercheurs, dont Christian Laville et Benedict Anderson, que l'étude du passé et la mise en récit de l'histoire a un rôle social à jouer dans la formation d'identités collectives $^{4}$ puisque la nation se construit aussi à travers le récit historique. Le cas du Canada français ne fait pas exception: l'histoire et l'étude de l'histoire occupent une place centrale dans l'élaboration d'un projet national. Si l'analyse reflète davantage la représentation du discours d'enracinement, comme certains auteurs l'ont proposé, la réflexion repose sur l'idée selon laquelle $l^{\prime}$ historiographie ${ }^{5}$ participe à la représentation du sentiment d'appartenance à un groupe, ou de la référence (Dumont, 1993). La référence chez Fernand Dumont est un concept selon lequel les membres d'un groupe intègrent dans leurs comportements et leur discours une identité partagée $e^{6}$. En ce qui a trait à l'analyse de notre corpus, il faut aussi souligner la superposition et la multiplication d'identités sur les territoires à l'étude. Il y a, au-delà de l'idée d'une origine commune et d'une histoire partagée (certes présentes dans le discours), une évolution et une fluctuation du sens d'appartenance, que ce soit pour des 
motifs géographiques ou générationnels. Il s'agit aussi, pour reprendre le concept évoqué par Marie Lebel, de situer au sein de ces représentations les «demandes de sens » (Lebel, 2010, p. $2,12,36)$ qui, aux fins de nos analyses, puisent leurs racines dans l'histoire et son territoire.

Puisque les deux publications étudiées paraissent assez hâtivement après la fondation des deux sociétés historiques, et qu'elles sont rédigées par des membres fondateurs, il importe de situer ces publications au sein de la prise de conscience historique que représente leur fondation. Au même titre que la fondation de revues savantes, l'acte de fonder une société d'histoire est une forme de prise de parole intellectuelle par le groupe, qui se voit par le fait même comme un groupe autonome distinct de la production intellectuelle existante. Il s'agit d'un «travail de groupe, d'équipe; s'y exprime la conscience d'un Nous qui prend la parole dans un milieu donné ${ }^{7} »$ (Fortin, 1993, p. 8-9). L'établissement de la SHFA et la SHSB et le contexte de fondation sont donc utilisés ici comme moyen d'analyse du discours d'enracinement par l'entremise de certains documents officiels de la fondation.

Les contextes de fondation de ces deux sociétés historiques ont certains points de convergence. Elles sont fondées au cours de la première des deux vagues de fondation de sociétés historiques de langue française en Amérique du Nord. Une analyse des dates de fondation de ces sociétés historiques semble indiquer que la première a lieu au tournant du XXe siècle et que la seconde s'est produite entre 1920 et 1940 (St-Pierre, 2015). Alors que la première vague semble plus intimement liée aux mouvements de migration canadienne-française, la seconde est davantage une expression du mouvement nationaliste canadien-français qui atteint en quelque sorte son apogée au cours des années 1930. Ces deux vagues sont distinctes l'une de l'autre puisqu'il ne semble pas y avoir de fondations de sociétés historiques de langue française en Amérique entre celles de Saint-Boniface en 1902 et celle de la Société historique trifluvienne en 1926 (Cadieux, 1948, p. 148). Qui plus est, la SHFA et la SHSB connaîtront toutes deux une relance de leurs activités dans les années 1930, participant ainsi, avec les sociétés historiques de la deuxième vague, à l'effervescence de l'histoire régionale de cette période. 


\section{Les mouvements de migration et l'émergence d'une conscience historique}

L'émergence dela production historiqueet de la conscience historiographique au Canada français coïncide avec l'émergence du projet national canadien-français. La seconde moitié du XIX ${ }^{\text {e }}$ siècle est aussi largement marqué par les mouvements de migration des Canadiens français qui quittent la vallée du SaintLaurent pour s'installer dans d'autres régions du continent. Bien que cette migration débute dans la première moitié du XIXe siècle, elle s'accélère après 1860 avant de culminer dans les années 1930. Le mouvement de migration le plus notable est de loin celui des Franco-Américains, qui amène près d'un million de Canadiens français à s'établir aux États-Unis (Roby, 2000, p. 11). Sous la direction d'une certaine élite locale (membres du clergé, notaires et médecins), ces Canadiens français se dotent d'institutions (principalement religieuses), dont la paroisse est le pivot. En Nouvelle-Angleterre, on retrouve une classe moyenne composée de prêtres, de médecins, d'avocats, de journalistes et de commerçants (Weil, 1990, p. 26). Bien que les Canadiens français migrent aussi dans d'autres régions des États-Unis, ils n'y forment pas les mêmes institutions et noyaux intellectuels qu'en Nouvelle-Angleterre, exception faite de la région du Détroit, fondée à l'époque du Régime français.

Les tentatives de rapatriement, qui ont lieu dans les années 1870, mènent à un échec (Roby, 2000 p. 106). Cet événement marque d'ailleurs un tournant dans l'histoire des Franco-Américains.

Vers 1880, un moment de l'histoire des Canadiens français de Nouvelle-Angleterre se termina. L'époque « héroïque » des premières communautés, des bâtisseurs, était passée. Les dirigeants canadiens-français avaient fait le choix des États-Unis contre le rapatriement. Les ouvriers canadiensfrançais avaient fait de même. Les structures s'étaient lentement mises en place. Tous les éléments étaient donc réunis au début des années 1880 pour que les Canadiens français cessent de rentrer au pays. Ce tournant était le prélude à la naissance d'une identité franco-américaine distincte de l'identité canadienne-française. (Roby, 2000, p. 115)

Étant donné l'importance de ce flux migratoire, on dénote une transformation des mentalités à l'égard des émigrés de la 
part de l'élite, cléricale et laïque. À la vue de ces Canadiens français quittant le Québec, on passe du désespoir à l'espoir d'une reconquête (parfois providentielle) du continent américain par la migration (Weil, 19990 p. 30). Dès la fin des années 1880, l'élite canadienne-française délaisse les jugements xénophobes entourant les mouvements migratoires des Canadiens français vers les États-Unis (Roby, 2000, p. 126) et le réseau paroissial passe de 86 paroisses nationales en 1891 à 202 en 1902, sans compter les 101 missions (Roby, 2000, p. 145). Ce sens nouveau donné à la migration sert d'inspiration aux auteurs et aux historiens qui s'inscriront dans la mentalité d'une reconquête du territoire par la migration, qu'ils verront comme un acte providentiel. La représentation du territoire historique chez ces historiens, qu'ils soient laïcs ou clercs, en est largement influencée par cette image de la reconquête.

Du côté de l'Ouest canadien, le mouvement de migration s'opère différemment. Bien qu'il s'apparente à celui qui pousse les Canadiens français à migrer vers le Sud, il s'en distingue sur plusieurs points. La migration vers l'Ouest ne s'arrime pas à l'urbanisation, et le rapport au majoritaire anglophone n'est pas du tout le même. Comme l'explique Frenette, la traite des fourrures avait déjà attiré de nombreux Canadiens français et « donné naissance [...] au peuple métis. » (Frenette, 1998, p. 86). En 1812, Thomas Douglas, mieux connu sous le nom de Lord Selkirk, fonde la colonie de la rivière Rouge suite à l'attribution de $300000 \mathrm{~km}^{2}$ par la Compagnie de la Baie d'Hudson. Par la suite, le clergé s'y installe pour convertir ou christianiser les populations autochtones et métisses tout en cherchant à rediriger le flux de population du Québec vers cette région. Il importe de noter qu'à l'inverse des États-Unis, le clergé ne s'oppose pas aux mouvements de migration vers l'Ouest, bien au contraire (Frenette, 1998, p. 88). L'Ouest est d'ailleurs central aux efforts de rapatriement de Canadiens français ayant migrés vers les États-Unis. Comme l'explique Robert Painchaud, si on cherche aussi à prévenir les départs vers les États-Unis en recrutant les colons vers l'Ouest, on assiste également à une véritable tentative de rapatriement (Painchaud, 1986, p. 130). Dans la période qui s'échelonne de 1874 à 1888, le rapatriement s'organise et devient « la méthode la plus acceptable de recruter des colons canadiens-français pour le Manitoba, puisque leclergé et les chefs politiques du Québec s'opposaient à tout programme 
qui pourrait encourager un dépeuplement additionnel de leur province» (Painchaud,1986, p. 130). La situation économique défavorable en Nouvelle-Angleterre semble fournir de l'eau au moulin, mais avec la reprise économique, à laquelle s'ajoute « le sentiment que les Canadiens pouvaient très bien être loyaux à leurs origines tout en acceptant la citoyenneté américaine » (Painchaud, 1986, p.135), les efforts de rapatriement s'estompent. À compter de 1887, elle est du ressort des missionnaires colonisateurs subventionnés par l'État, qui misent autant sur la prévention dans les campagnes québécoises que le recrutement de Canadiens français aux États-Unis. La colonisation de l'Ouest ne se limite toutefois pas aux tentatives de rapatriement, mais c'est surtout dans cette optique qu'elle est explorée. De plus, elle ne peut qu'être comparée aux mouvements de migrations vers les États-Unis puisqu'elles se produisent simultanément et que pour des raisons structurelles, notamment la distance et le coût du voyage, l'Ouest demeure moins attrayant que les villes manufacturières et industrielles de la Nouvelle-Angleterre ou encore du Mid-West américain (Frenette, 1998, p. 86-87). Qui plus est, Frenette explique qu'après la pendaison de Louis Riel en 1885, l'élite canadienne-française du Québec, jusque-là « fort tiède $[\ldots]$ s'oppose carrément, maintenant, à la colonisation d'une région qu'on juge gagnée aux Canadiens anglais et hostile aux Canadiens français » (Frenette, 1998, p. 87).

Si cette vague de migration vers l'Ouest sème de nombreuses petites communautés de langue française sur un vaste territoire, c'est autour de Saint-Boniface que naît la production historique dans l'Ouest canadien. Étant le siège épiscopal autour duquel gravite l'élite intellectuelle, Saint-Boniface est doté d'institutions centrales, comme son collège éponyme, fondé en 1855 et incorporé en 1871 (Pelchat, 2010). Au XIX siècle, SaintBoniface est donc un véritable pivot pour les Canadiens français de l'Ouest, de même qu'un centre intellectuel important qui se dote rapidement d'institutions. Elle devient un terreau fertile pour la production historique dès 1843 avec une publication de nature autobiographique de Mgr Norbert Provencher, qui sera suivie des livres de Mgr Taché, son successeur, parus en 1866 et 1869. C'est cependant davantage par les publications de l'abbé Dugas, notamment La première canadienne, (1833) que s'entame un discours historiographique plus substantiel. 


\section{Les sociétés historiques}

Dans cette prise de conscience historique, il importe de noter la place qu'occupent les sociétés savantes. Les sociétés savantes, dont les sociétés historiques, émergent un peu partout en Amérique à compter du début du XIX ${ }^{e}$ siècle. La première société savante de langue française, c'est-à-dire la Société littéraire de Québec, est fondée en 1809 (Bernatchez, 1981, p. 179). Telle que nous l'avons déjà précisé, il existe deux vagues successives de fondations de sociétés historiques en Amérique française, la première voyant le jour au tournant du $X X^{e}$ siècle et la seconde s'échelonnant de 1920 à 1940 (St-Pierre, 2015). Si les deux sociétés historiques à l'étude sont fondées au tournant du siècle, leurs contextes de création respectifs nécessitent de brèves descriptions puisqu'ils ont une incidence sur le rapport à l'Autre. À l'époque où l'on fonde la SHFA, on note trois facteurs contribuant à l'essor des Canadiens français des États-Unis : le réseau d'institutions, les sociétés nationales, qui permettent la formulation de revendications et de projets collectifs, et l'élite, composée de membres du clergé, de professionnels et d'hommes d'affaires (Bock, 2002, p. 182). Regroupés autour de ces institutions, les Canadiens français des États-Unis se représentent généralement comme des membres de la nation canadienne-française, du moins dans la seconde moitié du XIXe siècle. $C^{\prime}$ est au tournant $\mathrm{du} X X^{\mathrm{e}}$ siècle que le rapport à la nation se complexifie. Lorsque le mouvement de migration vers les États-Unis s'estompe, le fossé entre les Canadiens français du Québec et les Franco-Américains se creuse. De plus, aux ÉtatsUnis, on perçoit souvent les catholiques comme une menace et l'idée d'une double allégeance pose problème (Bock, 2002, p. 186), d'où l'importance de témoigner de son « américanité ». $\mathrm{Au}$ cours de son histoire, la SHFA se distingue des autres sociétés historiques de langue française du continent sur deux points. D'une part, il y a l'ambigüité de sa "terre ancestrale", qui se manifeste par une double, voire une triple allégeance identitaire - à la France, au Canada et aux États-Unis. D'autre part, il y a la présence de conférenciers illustres qui s'explique par le lien étroit qu'entretient la SHFA au Consulat de France et aux grandes universités américaines, dont Harvard, située à Boston, ville-hôte de la majorité des conférences de la SHFA (SHFA, 1939). 
Cette tension identitaire marque les Franco-Américains et $\mathrm{s}^{\prime}$ inscrit dans un contexte de fragilisation des liens avec le Canada français. Michel Bock précise que pour Groulx, c'est l'indifférence du Québec qui en est la cause (Bock, 2002, p. 192). Toutefois, la question est davantage complexe puisque c'est le rapport même à la nation canadienne-française, voire même à l'identité canadienne-française, qui se redessine. Comme l'expliquent Sylvie Beaudreau et Yves Frenette : «On se réclame encore du Canada français, mais on essaie de se donner une identité propre, notamment en adoptant le nom fort révélateur de "Franco-Américain" " (Beaudreau et Frenette, 1995, p. 240). La SHFA, fondée en 1899, en est un bel exemple. Selon Sénécal, la transition du vocable Canadien français à Franco-Américain s'exerce assez subitement : «En moins de dix ans, on assista à la métamorphose des Canadiens aux États-Unis, vocable presque universel pour désigner les expatriés québécois et acadiens, en Franco-Américains » (Sénécal, 1992, p. 209²). Cette redéfinition identitaire influence la production historique, et c'est cet aspect de la question que j'explore. Au cours des années 1899 à 1933, les historiens célèbrent les communautés francophones de la Nouvelle-Angleterre par le biais de monographies de paroisses ou d'historiques d'associations dans lesquels ils soulignent "les réalisations du groupe [afin de] conserver les traits de leurs ancêtres : langue française, religion catholique et valeurs traditionnelles » (Beaudreau et Frenette, 1995, p. 239-240). Cette identité passait par la France, surtout du côté de l'intelligentsia qui « avaitbesoin du prestige et du caractère 'antique' de la grandmère patrie pour rehausser le sien dans une nation américaine qui devenait de plus en plus cosmopolite» (Beaudreau et Frenette, 1995, p. 239-240). Bon nombre d'auteurs qui marquent de cette période se regroupent d'ailleurs autour de la SHFA. Généralement issus des professions libérales, les fondateurs de la Société ont pour objectif dans leur charte le désir d'étudier l'histoire des États-Unis et d'y préciser le rôle des descendants de race française dans la trame narrative de l'histoire du peuple américain (SHFA, 1939). Il s'agit d'un tournant puisque « [p]our la première fois, une institution canadienne des Etats-Unis à envergure nationale incorporait l'appellation franco-américaine à son nom et faisait référence à la race française dans son manifeste » (Sénécal, 1992, p. 212). Sénécal ajoute : «L'identité 
franco-américaine allait se fixer profondément dans les esprits et les cœurs nationalistes » (Sénécal, 1992, p. 212).

La Charte de la Société historique Franco-Américaine semble indiquer que le regard de la SHFA embrasse principalement les États-Unis (SHFA, 1939) et la société semble ainsi jouer un rôle de porte-parole vis-à-vis la majorité. L'un de ses fondateurs, J. Arthur Favreau, publiera quelques textes en anglais qui ont comme public cible les Américains dont le plus important est sans doute sa brochure intitulée The Chinese of the Eastern States : With the compliments of the Société Historique Franco-Américaine (Favreau, 1925). Les textes en anglais ont souvent comme objectif de faire valoir la présence des Franco-Américains sur le territoire au moyen de justifications historiques. L'article publié en 1909 dans The Catholic Encyclopedia, que cosignent J.L.K Lapalme, David Lavigne et J. Arthur Favreau, trois membres fondateurs de la Société historique franco-américaine, témoigne du rôle que jouent les fondateurs de la société, de même que de la place qu'occupe la Société parmi les lettrés de la NouvelleAngleterre. On y retrouve d'ailleurs dans le texte anglais à l'étude, une description de la Société historique qui témoigne de la place qu'elle accorde aux Franco-Américains dans l'ensemble des États-Unis. Les auteurs décrivent la SHFA en reprenant les grandes lignes de la Charte. Ils ajoutent :

With this end in view this society has met regularly twice a year since its organization. Noted American historians and writers, as well as several from France and Canada, have delivered before it addresses which have contributed in no slight measure to enrich the store of French American historical literature. (Favreau et al., p. 273)

Les objectifs de la Société historique sont ici clairement explicités: il s'agit d'écrire l'histoire des Franco-Américains afin de l'intégrer dans la grande trame narrative de l'histoire des États-Unis. Par la rigueur de la discipline historique, on s'engage à dresser une étude historique systématique de l'histoire des États-Unis qui permettra de faire valoir le rôle joué par la « race » française dans l'évolution et la formation du peuple états-unien.

La fondation de la SHSB est aussi largement influencée par son milieu. Le contexte de migration vers l'Ouest canadien 
que nous avons présenté dans les pages précédentes, témoigne de certaines distinctions qui influencent le rapport à l'Autre. On retrouve dans l'Ouest, entre autres choses, un mode de vie plus proche de la réalité agricole qui s'éloigne de celui de salarié à l'usine. On se côtoie, sans pour autant vivre au quotidien sous le regard de cet Autre qui prend la forme du superviseur ou du patron.

Dans le cas de la SHSB, l'enracinement passe par le droit de " premier venu » par la présence française sur le territoire et on retrouve des liens importants avec l'histoire de l'exploration par les Français. Il n'est donc pas surprenant de noter que c'est à La Vérendrye que revient le statut de « héros officiel » de la société. La fondation de la SHSB remonte à une expédition sur l'île au Massacre pour tenter d'y retrouver les ossements du héros français. Non seulement a-t-on justifié l'expédition des fondateurs en évoquant sa mémoire, La Vérendrye fera aussi l'objet d'une publication de la SHSB en 1911. Il s'agit d'une publication des manuscrits de cet explorateur dont le juge Prud'homme, auteur prolifique et membre de la Société, signe la préface (Prud'homme, 1911). Prud'homme avait aussi publié un texte portant sur La Vérendrye quelques années auparavant, dans les mémoires de la Société Royale du Canada (Prud'homme, 1905). Quelques années plus tard, sa publication de 1905 sera traduite en anglais. C'est ce texte qui fera l'objet d'une analyse dans les paragraphes qui suivent. La présence de La Vérendrye sur le territoire qui correspond au Manitoba actuel est un élément crucial des recherches et des publications de la Société et sa place dans le discours d'enracinement est évidente.

Dans le cas des deux sociétés, il importe aussi de noter la place importante de la religion et de l'Église dans le discours. Le groupe d'appartenance, que ce soit les Franco-Américains ou les Canadiens français de l'Ouest, peut se revendiquer de cette grande appartenance qu'est la religion catholique. On évoque ainsi les mœurs, notamment dans le texte de la SHFA où l'on précise que certains Canadiens français migrent aux États-Unis pour le luxe, mais qu'il s'agit, pour la majorité, d'une mission providentielle dont l'importance sera révélée à l'avenir (Favreau, et al., 1909, p. 272). Du côté de La Vérendrye, la publication de Prud'homme le présente comme ayant été responsable 
d'apporter les lumières de la civilisation aux contrées sauvages, revendiquant ainsi une appartenance qui dépasse largement la France - La Vérendrye représente la civilisation. Qui plus est, la présence de francophones et de leurs institutions dans l'Ouest lui est tributaire.

\section{L'enracinement de soi et la représentation historique pour l'Autre}

Les deux textes rédigés en anglais s'inscrivent dans une production plus globale de textes historiques rédigés par les deux sociétés historiques. Dans le cas de la SHFA, les publications, conférences et autres textes sont colligés dans un ouvrage qui souligne le $40^{\mathrm{e}}$ anniversaire de la société. On y répertorie 91 conférences : seules trois d'entre elles ont été données en anglais et une seule a été prononcée dans les deux langues (SHFA, 1939) $)^{9}$. S'y ajoutent certains textes, dont l'article d'encyclopédie à l'étude, rédigé par les membres fondateurs de la SHFA bien qu'il ne soit pas répertorié dans l'ouvrage du 40e (SHFA, 1939). Dans le cas de la SHSB, le texte à l'étude est une traduction de la version en langue française parue dans les publications de la Société royale du Canada. (Prud'homme, 1905). Il s'agit de la seule publication d'envergure de langue anglaise répertoriée. Un autre texte de langue anglaise est publié en 1913 par la SHSB afin de solliciter des dons pour l'érection d'un monument en l'honneur de La Vérendrye (Prud'homme et Lamy, 1913) ${ }^{10}$.

Si la langue anglaise ne figure pas de façon dominante comme langue d'usage dans la production historique de la SHFA, il n'en demeure pas moins que l'on s'intéresse aux questions linguistiques lorsque l'on mise sur l'américanité des Franco-Américains. Dans le texte à l'étude, intitulé French Catholics in the United States, les auteurs précisent:

The question of language stood in the way from the very beginning. The French Canadians, though willing to become staunch Americans, did not know the English language, and even when they had learned it, they still preserved a strong attachment for their mother tongue. [...] Even to-day this problem is not adequately solved. It was feared at the beginning, as it is feared now in some quarters, that to grant to the French Canadian immigrants priests of their own tongue and nationality would encourage them to form a sort of state within the state, thereby causing great harm to the nation as 
a whole. Time has shown the fallacy of that argument. The patriotism of the French American element is undisputed. They possess the sterling civic qualities desirable and necessary to promote the best interests of the republic. As a matter of fact, the French Canadian immigration has created no new state in the state; and the French Americans have willingly learned the English language while remaining as closely attached as ever to their mother tongue, in which they see the best safeguard of their faith ${ }^{11}$. (Favreau, et al., 1909, p. 272)

Les références au patriotisme et aux intérêts voués des Franco-Américains pour la République sont des thèmes récurrents dans le texte. Si l'objectif principal des auteurs est de présenter les catholiques de langue française aux ÉtatsUnis - il s'agit d'un article dans The Catholic Encyclopedia, après tout - on constate, dès les premières lignes, que l'objectif sousjacent est plus complexe. Les auteurs cherchent premièrement à démontrer la présence des Franco-Américains, leur importance, et leur primauté sur le territoire. Parsemé de statistiques de nature démographique, le texte note non seulement la place que les Franco-Américains occupent par leur nombre, mais aussi leur intégration. Ainsi, on insiste sur le fait que ce sont les immigrants canadiens-français permanents qui sont les plus nombreux en comparaison aux immigrants Canadiens français de passage. «It is these permanent Franco-American immigrants who have organized parishes and parochial schools, erected churches and convents, and now constitute the laboring power par excellence in all the industries of New England » (Favreau, et al., 1909, p. 272).

Incontournables, donc, par leur nombre et leur présence continue, les Franco-Américains sont surtout décrits pour leur rôle et leurs nombreuses contributions. Les auteurs reviennent d'ailleurs à plus d'une reprise sur leur rôle économique. Après avoir énuméré leurs multiples contributions dans le monde religieux, les auteurs ajoutent:

Besides their religious work, vast and praiseworthy as it is, the French Canadian immigrants have also displayed industry and activity in other walks of life, and in their closer relations with their fellow-citizens they have shown qualities and traits found only in the best of citizens. (Favreau, et al., 1909, p. 272) 
Ou encore, les auteurs précisent que selon des spécialistes de l'économie, les Franco-Américains constituent, « as labourers and artisans, the most solid pillar of industry in New England » (Favreau, et al., 1909, p. 272).

Les actions et les contributions des Franco-Américains passent souvent par leur histoire qui, selon les auteurs, est très peu connue de la population américaine. Les auteurs retracent donc les actions dans le temps en utilisant, comme points de référence, les moments clés de l'histoire américaine. On tient donc compte du public cible états-unien, de sorte que les actions du groupe franco-américain sont davantage enracinées dans la trame narrative de l'histoire américaine que de celle du Canada, témoignant ainsi de leur américanité. L'un des passages les plus marquants à cet effet porte sur le rôle des descendants français dans l'armée de Washington dans lequel on peut lire: "The immigration of French Canadians to the United States began before the War of American Independence (1775-1783). French Canadians had then already immigrated to New England, and we find them in large numbers in the armies of Washington " (Favreau, et al., 1909, p. 272). On ajoute que ces derniers, pour leur rôle dans la guerre d'indépendance, sont passibles d'une charge de trahison au Canada et que le Congrès américain, en guise de reconnaissance, leur donne des terres sur les rives du lac Champlain où l'on retrouve encore certains de leurs descendants. En dépit de cela, et «[n]ot withstanding the fact that they had at that time but few organized parishes, the French Canadians were here in sufficient numbers during the war to furnish 40,000 soldiers to the Union » (Favreau, et al., 1909, p. 272). Leurs contributions à la vie civile et militaire américaine sont donc importantes.

Si la trame narrative à laquelle on adhère dans le texte est l'histoire états-unienne, on retrouve néanmoins certaines références à l'histoire canadienne-française qui surviennent plus tard dans le texte. Les seuls événements historiques canadiens qui sont abordés directement par les auteurs semblent avoir comme objectif d'illustrer l'appartenance à part entière des Franco-Américains aux États-Unis. À titre d'exemple, lorsqu'on parle du mouvement de migration des Canadiens français vers les États-Unis. 
This migration was called at the time «the desertion of the Fatherland». But those who spoke thus were forgetful of the historical fact, that the French of America have from the very beginning felt perfectly at home in the whole northern part of the continent, on the soil of which their missionaries, their coureurs des bois, explorers, and warriors have left their footprints broadcast. ${ }^{12}$.( Favreau, et al., 1909, p. 272)

Les auteurs précisent d'ailleurs qu'en dépit de l'opposition, des centaines de milliers de Canadiens français quittent les fermes « to adopt the more arduous life of the New England factories and the various industries of the Western States » (Favreau, et al., 1909, p. 272). Dans cette citation, qui évoque de façon très directe l'histoire des descendants français en Amérique du Nord, la référence au territoire historique est on ne peut plus claire. Qui plus est, le territoire d'origine des Canadiens français ne se limite pas au territoire actuel du Canada. Le vocable «French of America » semble aussi judicieusement choisi, tout comme les références aux missionnaires (dans une encyclopédie catholique), aux coureurs de bois (archétype de la Nouvelle-France), aux explorateurs et aux guerriers, faisant ainsi écho aux quelques références quant au rôle des Canadiens français dans les efforts de guerre de leur terre d'accueil.

Du côté de la SHSB, la publication de langue anglaise est une traduction d'un texte ayant été publié par Prud'homme quelques années auparavant dans les Mémoires de la Société Royale du Canada. Une comparaison de l'introduction, de la préface, ainsi que la première section du texte, qui sert d'introduction générale, permet de déceler des différences importantes qui sont attribuables à la langue de production et au public cible. Dès le début de introduction, on peut lire :

Western Canada owes a deep debt of gratitude to two great men, whose achievements will endear them forever to future generation. La Vérendrye, the discoverer and Selkirk, the father of the first colony of the West, by their unfaltering perseverance and noble qualities of heart and mind, have opened up the Wild West to Christian civilization. (Prud'homme, 1916, p. i)

Si Prud'homme évoque l'importance de reconnaître le rôle de La Vérendrye dans d'autres publications, cette référence à Lord Selkirk est entièrement absente de la version française du 
texte (Prud'homme, 1905). Prud'homme poursuit en précisant ses objectifs:

I have tried in this work, to outline the chief characteristics of the Discoverer and pay to his memory the tribute of admiration which it so justly deserves. Due justice has already been done by historians to the noble Lord who followed the Discoverer and revealed to the world the immense resources of our fertile land ${ }^{13}$. (Prud'Homme, 1916, p. i)

S'ensuit un bref hommage à Selkirk. Il est intéressant de noter qu'en dépit du commentaire de l'auteur à l'égard de la reconnaissance de Selkirk par de nombreux historiens, Prud'homme choisit néanmoins de lui consacrer quelques lignes. Qui plus est, la référence à Selkirk permet de situer les deux personnages dans la chronologie et la trame narrative de l'Ouest, de sorte que Selkirk succède à La Vérendrye en termes de présence sur le territoire historique. Il s'agit d'une référence directe à la place du "premier venu», qui est centrale au discours d'enracinement dans les prairies canadiennes. Dans les lignes qui suivent le bref hommage à Selkirk, Prud'homme écrit:

He richly deserves to be considered as a great benefactor of our country and his name should forever be associated with that of La Vérendrye in our gratitude. True it is, that the French Canadians have a special claim on La Vérendrye and the English Canadians on Selkirk, but these two great men, cannot be held within the compass of only one nationality. They are the glory of the West, and the fathers of that part of Canada. (Prud'homme, 1916, p. i-ii)

Il poursuit cette lancée, qui place les deux hommes sur un pied d'égalité, en exhortant tous les Canadiens à se familiariser avec les deux personnages.

All Canadians should learn their name, study their life and try to imitate their spirit of sacrifice for the love of their country. We should therefore all join in crowning their glorious brows with wreath of Laurels so dearly gained by their patriotism. Their careers are worthy of envy and emulation. They were nation builders by their indomitable spirit and dogged pertinacity. (Prud'homme, 1916, p. ii)

Prud'homme explique : 
We will find in it, precious lessons for the races who have followed the footsteps of La Vérendrye and inspiring examples for all inhabitants of the West. It is only by mutual respect for the laws of justice and true kindness for each other, that we may expect to lay the lasting foundations of a nation great, strong, prosperous and content and to cement its component parts in brotherly love and harmony by indissoluble ties, under the British flag. (Prud'homme, 1916, p. ii)

Lorsqu' on se penche sur l'introduction dans le corps du texte, on constate rapidement les éléments clés sur lesquels Prud'homme souhaite attirer l'attention de ses lecteurs. Dès les premières lignes, il évoque un autre personnage connu -Jacques Cartier - avant d'énumérer les nombreuses contributions et qualités de La Vérendrye;

This great discoverer was the Jacques Cartier of the North-West. He was the first white man to dip paddle in the waters of the Red or Assiniboine rivers, and the first whose voice singing the merry Canadian ditties was echoed through the solitudes of these unexplored lands. He brought to the tribes whom he visited, the sweet language of France, the light of the Faith ever advancing on his far reaching expeditions in company with Jesuit missionaries who sowed in this virgin soil the first seeds of the Gospel. (Prud'homme, 1916, p.5)

Le statut de "premier venu» de La Vérendye sur le territoire est omniprésent dans la citation ci-dessus. De plus, lorsqu'il énumère les qualités du personnage, il insiste sur le fait qu'ils'agit de caractéristiques qui lui permettent de se distinguer des autres explorateurs, des caractéristiques «[that raise] him above the other discoverers of his time » (Prud'homme, 1916, p. 6), notamment son courage à toute épreuve, sa foi et son caractère pieux, sa capacité de raisonnement, sa sagesse et ses capacités stratégiques. Il est intéressant de noter que, pour Prud'homme, le rôle de La Vérendrye dépasse largement celui de commerçant, ses véritables ambitions n'étant pas que pécuniaires;

La Vérendrye proposed to plant in the virgin soil of our prairies the seed of lasting institutions; accordingly he took care to establish them on a strong foundation and brought with him missionaries who at the dawn of the discovery of the west would establish the faith. We must not forget this grand purpose of La Vérendrye, a purpose 
so full of a weighty lesson. Human passions may later on adulterate this first seed and prevent for a time its full development, but the divine principle which is its source and the reason of its fecundity will henceforth ensure its duration and abundant fruition.

Great minds appreciate the importance of laying the foundation of a colony in this way. Western Canada at the time of its discovery had in its heart the teachings of the true Gospel. Catholicism imprinted on its forehead the seal of that moral grandeur which makes its present strength and its future hope. (Prud'homme, 1916, p. 1011)

Ainsi, Prud'homme établit un lien direct entre La Vérendrye comme personnage clé du passé Canadien français dans l'Ouest, et comme symbole de rigueur, de force et d'espoir pour le présent et l'avenir. Ce passage permet de situer encore plus clairement le sens que l'on cherche à donner à cette histoire et à l'enracinement de la population Canadienne française sur le territoire historique qu'on évoque. Les personnages marquants du passé servent à ancrer la population actuelle dans le territoire par une référence au lien qui les unit. On mise ainsi sur le passé pour mieux expliciter les valeurs actuelles qui s'y rattachent (en l'occurrence, les valeurs religieuses), le projet qui en émane et le gage d'avenir.

\section{Conclusion}

Cet article analyse la représentation du territoire historique pour l'Autre dans les publications de deux sociétés historiques en milieu minoritaire. La place qu'occupe le discours d'enracinement, rédigé par soi pour l'Autre, est analysée par l'entremise de deux textes produits en anglais par la SHFA et la SHSB. Le discours d'enracinement proposé par ces deux textes se juxtapose au discours qui entoure la fondation des deux sociétés historiques. Le moment de la fondation des deux sociétés historiques, ou l'acte fondateur, est présenté ici comme élément nous permettant de comparer le discours d'enracinement tel qu'il est élaboré par les fondateurs et celui présenté dans les deux publications de langue anglaise. Le moment de la fondation nous permet de situer l'élaboration d'un territoire historique au moment de l'émergence d'une conscience historique dans ces deux régions. Dans le cas de la SHFA et SHSB, ce sont des membres fondateurs de sociétés qui signent les deux textes anglais. Le discours d'enracinement, 
tel qu'il est représenté au moment de la fondation, devient donc un point de comparaison important afin de déterminer la façon dont la rédaction pour l'Autre, qui est majoritaire et anglophone, intervient sur le discours. De plus, dans le cas de la SHSB et du texte de Prud'homme, il en existe une version française (antérieure à la version anglaise), qui sert d'outil d'analyse comparatif.

Si certains grands traits du discours d'enracinement demeurent, force est de constater que le discours d'enracinement est quelque peu modifié dans les deux publications de langue anglaise. Dans les deux cas, le sujet qui est abordé n'est pas étranger à la société historique et on en retrouve des traces ailleurs, notamment dans le mandat de la société ou dans les publications de langue française. Du côté de la SHSB, il s'agit d'examiner la place prépondérante de La Vérendrye et de son rôle comme "premier venu » sur le territoire. Du côté de la SHFA, il s'agit d'examiner l'allégeance des Franco-Américains à leur terre d'accueil, les États-Unis. D'un côté comme de l'autre, l'élément central au discours véhiculé par la société demeure. Les nuances sont néanmoins présentes et témoignent d'une certaine hybridité dans la construction identitaire. Ce qui marque dans cette analyse de la transformation du discours d'enracinement, c'est la composante comparative. Le discours d'enracinement intègre donc d'autres événements et d'autres éléments clés qui comparent le groupe d'appartenance et l'Autre. Les références à Selkirk dans le texte de Prud'homme, de même que les multiples références à l'histoire américaine dans le texte de la SHFA en sont des exemples notables. Du côté de la SHFA, on mise beaucoup sur les actions des Canadiens français en terre d'accueil après leur immigration. On accorde, par ailleurs, une place importante à leurs actions militaires et au rôle qu'ils jouent dans des événements importants non pas la trame narrative historique du Canada français, mais bien celle des États-Unis.

Les sociétés historiques servent de témoin intéressant de la représentation de l'histoire par une certaine tranche de l'élite. En analysant la production historique pour l'autre, on remarque que les distinctions qui s'opèrent entre le discours d'enracinement présenté par les auteurs lorsqu'ils s'adressent à leur groupe d'appartenance, par rapport au discours qui est 
présenté pour l'Autre, permettent de mieux appréhender le rôle de l'histoire et de la représentation historique dans l'élaboration de discours sur le passé et sur l'identité, celle que l'on conçoit pour soi et celle que l'on souhaite projeter.

\section{NOTES}

1. Je tiens à remercier mes directeurs, Thomas Wien et Michel Bock de même que le département d'histoire de l'Université de Montréal, le CRCCF et le CRSH pour l'octroi de bourses de recherche. Un merci particulier aux organisatrices de la journée d'étude et rédactrices invitées du présent numéro, Isabelle $C$. Monnin et Geneviève Piché de même qu'aux évaluateurs anonymes pour la relecture de mon texte et leurs précieuses suggestions.

2. De la moitié du XIXee siècle jusqu'à environ 1930, plus de 900000 Canadiens français originaires du Québec quittent la province en direction des États-Unis (Anctil et Frenette, 2012). Dans le cas des Franco-Ontariens, «la population des petites communautés francophones de l'Ontario ne s'agrandit pas avant les années 1840, lorsque la population en accroissement rapide du Québec commence à se déverser dans l'Est de l'Ontario, puis près 1880, dans la région de Sudbury et enfin, au tournant du siècle, dans les « ceintures d'argile » du Nord (Haileybury-Hearst) » (Savard, 2012).

3. Voir, par exemple, les travaux de François Weil, Yves Roby, Yves Frenette, Marcel Martel, Gaétan Gervais, Michel Bock.

4. Notons les travaux de Steven Prickett, Claude Raffestin, Roger Chartier, Daniel Nordman, Peter Sahlins, Kathleen Wilson, Frederick Cooper et Rogers Brubaker.

5. Chez Dumont, la référence s'exprime de par l'idéologie, la littérature, et l'historiographie.

6. Fernand Dumont, Genèse de la société québécoise, Montréal, Éditions du Boréal, 1993, 393 p.

7. Alors que Fortin s'intéresse à la fondation de revues savantes, nous adoptons son modèle pour étudier l'acte de fonder les sociétés historiques, notamment la Société historique du Nouvel-Ontario dont il est question dans cet article.

8. «Au tournant du siècle, à l'époque euphorique où l'élite canadienne-française envisageait un avenir grandiose qui préconisait la conquête de la Nouvelle-Angleterre par l'ancienne Nouvelle-France, les apologistes nationalistes se mirent à désigner les leurs en terre promise sous le vocable de Franco-Américains. Ce baptême se fit des plus soudains. En 1892, Monseigneur Racine, évêque de Sherbrooke, rédige un mémoire sur la situation des 
«Canadiens français aux États-Unis » en 1913, en parlant des mêmes ouailles, le Père Denis-Michel-Aristide Magnan évoque les «Franco-Américains » en Nouvelle-Angleterre et la " race française aux États-Unis ». En 1888, Benjamin Lanthier fonde le journal Le Canado- Américain d'Holyoke (Massachusetts); en 1908, Jean-Léon-Kemmer Laflamme lance La Revue franco-américaine, la première publication sériée, publiée par un immigré canadien, à porter ce nom composé. En moins de dix ans, on assista à la métamorphose des Canadiens aux États-Unis, vocable presque universel pour désigner les expatriés québécois et acadiens, en Franco-Américains » (Sénécal, 1992, p. 209).

9. Puisqu'il n'y a que 44 textes complets, il est possible que certaines conférences dont le titre est en français étaient, dans les faits, présentées en anglais ou avec des sections en anglais.

10. Le projet d'ériger un monument en l'honneur de La Vérendrye occupe une place importante à la SHSB depuis 1912. Ce n'est qu'en 1938 que le monument verra le jour.

11. C'est moi qui souligne.

12. C'est moi qui souligne.

13. C'est moi qui souligne.

\section{BIBLIOGRAPHIE}

ANCTIL, Pierre, revue par Yves FRENETTE (2012), «FrancoAméricains ", L'Encyclopédie Canadienne, http://www. thecanadianencyclopedia.ca/fr/article / franco-americains, page consultée le 12 mars 2017.

ANDERSON, Benedict (2006), L'imaginaire national: Réflexions sur l'origine et l'essor du nationalisme, trad. de l'anglais par DAUZAT, Pierre-Emmanuel, Paris, Éditions de la découverte, $224 \mathrm{p}$.

BEAUDREAU, Sylvie et Yves FRENETTE (1995) « Historiographie et identité collective en Amérique française : le cas des élites francophones de la Nouvelle-Angleterre, 1872-1991 » dans LANGLOIS, Simon (dir.), Identités et cultures nationales. L'Amérique française en mutation, Québec, Presses de l'Université Laval/CEFAN, p. 233-254.

BOCK, Michel (2002) " "Suicide de la race" ou "vocation apostolique"? La représentation des Franco-Américains dans l'Action française de Montréal (1917-1928) » dans WALLOT, JeanPierre, Construction identitaires et pratiques sociales: Actes du colloque en hommage à Pierre Savard tenu à l'Université d'Ottawa les 4,5,6 octobre 2000, Ottawa, Presses de l’Université d'Ottawa, p. 175-200. 
CADIEUX, Lorenzo, (1948), « Mémoire en vue d'un meilleur rendement de nos sociétés d'histoire régionale ", Revue d'histoire de l'Amérique française, vol. 2, nº 1, 1948, p. 147-151.

DUMONT, Fernand, (1993) Genèse de la société québécoise, Montréal, Éditions du Boréal, 393 p.

FAVREAU, Joseph-Arthur, LAFLAMME, J. L. K., LAVIGNE, David E. (1909), "French Catholics in the United States », Catholic Encyclopedia, vol. VI, p. 271-277.

FAVREAU, Joseph-Arthur (1925), The Chinese of the eastern states: with the compliments of the Sociéte historique franco-américaine, Manchester, L'avenir national Pub. Co., 23 p.

FRENETTE, Yves (1998), Brève histoire des Canadiens français, Montréal, Boréal, 210 p.

LAVILLE, Christian (1985), «Minorités, compréhension mutuelle et usage de l'histoire », Cahiers de Clio, vol. 84, p. 17-33.

LEBEL, Marie (2010), Prises de parole et modes de l'engagement intellectuel dans le Nouvel-Ontario, 1970-1995, Thèse de doctorat (histoire), Université Laval, 494 p.

PAINCHAUD, Robert (1986), Un rêve français dans le peuplement de la Prairie, Saint-Boniface, Édition des Plaines, 303 p.

PELCHAT, Carole (2010), « Le collège universitaire de Saint-Boniface », L'Encyclopédie du patrimoine culturel de l'Amérique française [En ligne] Québec, page consultée le 1 ${ }^{\mathrm{er}}$ avril 2017.

PRUD'HOMME, Louis-Arthur (1905), « Pierre Gaultier de Varennes, sieur de La Vérendrye : capitaine des troupes de la marine, chevalier de l'ordre militaire de Saint-Louis, découvreur du nord-ouest, 1685-1749 », Société royale du Canada, Ottawa, p. 9-57.

(1911) «Documents sur la découverte du Nord-Ouest, SaintBoniface », Bulletin de la Société historique de Saint-Boniface, Vol I. $234 \mathrm{p}$.

(1916), Pierre Gaultier de Varennes sieur de la Vérendrye, captain of marines, chevalier of the military order of St.Louis, discoverer of the North-West, 1685-1749, Saint-Boniface, Historical Society of Saint-Boniface, 178 p.

PRUD'HOMME, Louis-Arthur et Denys LAMY (1913), A monument to LaVérendrye The Discoverer of the West, 1913, 19 p.

ROBY, Yves (2000) Les Franco-Américains de la Nouvelle-Angleterre: Rêves et réalités, Montréal, Septentrion. 526 p. 
ST-PIERRE, Stéphanie (2015), "Clercs et historiens: Le discours d'enracinement et la Société historique du Nouvel-Ontario (1942 à 1950) ", Études d'histoire religieuse, vol. 81, n 1-2, pages 59-79.

SAVARD, Pierre (2012) « Franco-Ontariens », L'Encyclopédie Canadienne, http : / / www.thecanadianencyclopedia.ca/fr/article/ franco-ontariens /, page consultée le 12 mars 2017.

SÉNÉCAL, Joseph-André (1992) «De «Canadiens français aux États-Unis « à "FrancoAméricains «: What's in a name? », Francophonies d'Amérique, pages 209-217.

SOCIÉTÉ HISTORIQUE FRANCO-AMÉRICAINE, (1939), Quarante ans de la Société historique franco-américaine, 1899-1939, Boston, Société Historique Franco-Américaine, 878 p.

WEIL, François (1990), Les Franco-Américains : 1860-1980, Paris, Belin, $254 \mathrm{p}$. 


\section{Commentaire de Nicole St-Onge}

(Université d'Ottawa)

Dans son article, Stéphanie St-Pierre analyse les contextes de diffusion et les messages véhiculés dans deux publications rédigées en anglais par des Sociétés historiques de langues françaises : la première, la Société historique franco-américaine (SHFA), est fondée en 1899 et la deuxième, la Société historique de Saint-Boniface, date de 1902. L'objectif proposé est de mieux saisir « la place qu'occupe la représentation du territoire historique et du discours d'enracinement dans l'étude de l'histoire de soi pour l' Autre, qui est majoritaire et anglophone ». St-Pierre constate que, bien que le discours d'enracinement perdure dans les deux publications examinées - comme il avait été véhiculé dans les versions françaises de ces deux mêmes textes qui prédatent leur publication en anglais - les textes ont tous deux été un tant soit peu modifiés pour rendre plus acceptable et " normale » une présence franco-catholique aussi bien sur le terroir étatsunien que dans l'Ouest canadien. Dans le texte de la SHSB, on intègre une mention louangeuse du comte Selkirk comme un des pionniers fondateurs de l'Ouest canadien au côté de La Vérendrye. En contrepartie, le texte de SHFA multiplie les mentions de l'apport des franco-catholiques aux évènements clefs de l'histoire américaine, surtout du côté militaire.

Le texte de Stéphanie St-Pierre est fascinant et suscite l'intérêt pour tous ceux qui s'intéressent au sujet. Le lecteur ne peut qu'espérer qu'une analyse plus poussée dans un texte plus long fasse partie des projets d'avenir de cette jeune historienne. Si oui, certains sujets pourraient être creusés avec profit. Par exemple, il nous semble que le contexte général dans lequel ces deux publications sont traduites et publiées dans «la langue de l'autre » pourrait être étudié en plus grande profondeur. Nous parlons ici du contexte à l'échelle nordaméricaine puisque des individus, des journaux ainsi qu'une vaste correspondance circulaient à cette échelle continentale au sein des élites religieuses et séculières franco-catholiques. La publication de la SHFA paraît en 1909 celle de la SHSB en 1916. Le compromis Laurier-Greenway limitant l'enseignement de la langue française au Manitoba date de 1896, l'abolition de la 
langue française dans les écoles publiques de la Saskatchewan et de l'Alberta est loi en 1905, de 1912 à 1926 les retombées du règlement 17 en Ontario - reflétant partiellement un combat de pouvoir entre Canadiens français et Canadiens irlandais en Ontario - se font sentir. Du côté américain, en 1916, la Louisiane française voit l'usage du français interdit dans les écoles et cinq en plus tard, en 1921, ce même état autorise uniquement l'usage de la langue anglaise dans sa nouvelle constitution. Plus près du chef-lieu de la SHFA, le Collège Assomption, fondé en 1904 à Worcester (Massachussetts), connaitra des rivalités et des tensions linguistiques franco-anglaises dès ses débuts. Cette liste fort sommaire explique peut-être en partie pourquoi les chefs de file de la SHSB et de SHFA se justifient dans la langue de Shakespeare - peut-être se sentent-ils assiégés et menacés? Si tel est le cas, quel impact ces sentiments ont-ils dans la rédaction de ces textes d'abord en français puis en anglais?

Une deuxième ligne d'enquête qui pourrait enrichir un texte plus long serait une analyse des nombreuses zones de silence qu'on remarque dans les textes. Comme Stéphanie StPierre explique clairement, le texte de la SHFA essaie de démontrer l'appartenance des Franco-Américains au terroir américain. Mais, c'est l'appartenance de seule une population très précise d'individus. En d'autres mots, seulement les immigrants d'origine canadienne-française ou leurs descendants sont ici dignes d'attention. On remarque par exemple dans le tableau reproduit dans la publication de la Catholic Encyclopedia démontrant la distribution des Franco-Américains aux ÉtatsUnis que seuls les francophones nés a l'étranger, ou les enfants nés de parents étrangers, sont énumérés. Donc, en Louisiane, par exemple, on ne compte que 1012 individus francoaméricains. Les Cajuns et les créoles franco-catholiques de la Louisiane, les vieilles populations francophones de la ville de St. Louis (Missouri) et de la vallée du Mississippi sont en général passées sous le silence - et ceci même si des centres tels St. Louis ou La Nouvelle-Orléans comprennent des élites laïques et religieuses qui fonctionnent toujours en français au début du vingtième siècle. Pourquoi donc y a-t-il ce désir, tant chez Favreau, que chez Laflamme, chez Lavigne et de la part de la Société histoire franco-américaine de se distinguer, ou du moins ne pas démontrer de liens étroits, avec ces populations qui partagent pourtant même langue et religion? Est-ce un relent de 
la guerre civile américaine? En d'autres mots, la perception que ces populations sont sudistes et historiquement esclavagistes amène-t-elle les Canadiens français à ne pas vouloir s'identifier avec le côté perdant dans ce conflit sanglant? Les FrancoAméricains regroupés par la SHFA veulent-ils projeter une image de nordicité forte et fière? Ou est-ce une réticence face à l'origine mixte de certaines de ces populations? À St. Louis comme à La Nouvelle-Orléans, plusieurs catholiques qui parlent français sont, partiellement du moins, aussi Afro-Américains. La montée du darwinisme social à cette époque influence-t-elle la rédaction du texte de la SHFA et la projection de « soi » qui s'y retrouve, à savoir que les auteurs se perçoivent comme étant plus comme les Américains anglos-protestants que les sudistes franco-catholiques? La même question se pose face au texte de Prud'homme. Dans la version anglaise, l'auteur évoque le comte Selkirk comme le fondateur de la colonie de la rivière Rouge et, donc, le descendant spirituel de La Vérendrye. Mais, un peu comme les auteurs franco-américains, Prud'homme passe sous silence la population de voyageurs canadiens-français et de leur descendance métisse qui prédatent l'arrivée de Selkirk et des colons écossais dans la vallée de la rivière Rouge. Le tout semble étonnant à un certain niveau si le but de l'exercice est de valider aux yeux de la majorité anglo-protestante le droit d'appartenance d'une population franco-catholique dans les Prairies canadiennes. La question se pose à nouveau: est-ce une question de " race " qui entre ici en ligne de compte? Catholique - certainement; francophone - fièrement; mais aussi, et peut être surtout - Blanc? Un peu différent, mais pas trop, de « l'autre » ciblé par ces publications?

Poursuivre les pistes de recherche proposées dans les paragraphes précédents serait fascinant - surtout dans le contexte actuel. Que l'on pense à la popularité de regroupements franco-américains tel le site Facebook Great Lakes FrenchCanadians \& Métis $^{1}$ ou encore, dans les Prairies canadiennes, aux nombreuses recherches généalogiques qui « prouveraient » que des individus dont les parents ou grands-parents se seraient définis comme "canayens" auraient en fait des origines métisses : le désir d'appartenir à un groupe «de souche » est toujours aussi présent. Cependant, le profil de ce groupe a évolué - un critère clef de nos jours semble justement être cette origine «mixte » (sans toutefois, et toujours de façon fascinante, 
être "africaine»). Pour beaucoup de descendants d'origine caanadienne-française, que ce soit dans les Prairies canadiennes ou aux États-Unis, la langue et la religion ne sont plus des repères identitaires valides. Pourtant le désir d'appartenir et de se distinguer de "l'autre » perdure. Contrairement au début du vingtième siècle, où langue et foi suffisaient amplement et que les différences perçues comme raciales étaient passées sous silence, de nos jours la langue et la religion sont seulement évoquées pour décrire les générations passées. Ce qui attire et tisse des liens (virtuels ou réels) pour plusieurs dans la population actuelle d'origine canadienne-française est une idée d'appartenance communautaire « métissée ».

\section{NOTE}

1. https://www.facebook.com/groups/FCCAGL/ 


\section{Réponse de Stéphanie St-Pierre}

Université de Montréal

Je tiens à remercier Nicole St-Onge pour sa lecture de mon article et ses précieuses pistes de réflexion. Dans son commentaire, l'auteure aborde plusieurs aspects des plus percutants en matière de recherche sur l'identité ou sur les identités et je suis très reconnaissante qu'elle ait accepté de commenter mon article. La question du métissage identitaire s'inscrit droit au cœur de mes préoccupations, tant personnelles que professionnelles. On retrouve, comme trame de fond du commentaire de St-Onge, un questionnement entourant la définition même du fait français dans les Amériques et de(s) identité(s) qui en découle. La multitude de visions de ce qu'est l'espace francophone nord-américain, la francophonie (ou les francophonies), les origines, et/ou l'appartenance, sème sur son passage autant de pistes de recherche potentielles. Afin de bien répondre aux divers commentaires et suggestions, je vais d'abord situer l'article dans son contexte de production plus large pour ensuite apporter quelques éléments de réponses et de précisions.

L'article s'inscrit dans le cadre de mes recherches doctorales qui portent sur la représentation du territoire historique et du discours d'enracinement dans les historiographies de langue française en milieu minoritaire produites entre 1845 et le début des années 1990. J'étudie cette évolution par l'analyse d'un corpus d'ouvrages historiques sélectionnés qui sont généralement rédigés par et pour les Canadiens français qui habitent ces territoires. Centrée sur la nation canadiennefrançaise, j'examine l'objet autrement, tant par l'espace que par le temps. Au cours des dernières années, on a écrit beaucoup au sujet de certaines régions de cette nation canadienne-française. Les études ciblées portant sur les Franco-Américains (Weil, 1990 et Roby, 2000) et les Franco-Manitobains (Painchaud, 1984) et autres groupes tels les Franco-Ontariens, les Fransaskois, les Acadiens, etc. se multiplient. On retrouve exceptionnellement quelques synthèses, dont celle d'Yves Frenette (Frenette, 1998). Mon étude cible donc un espace ou un territoire plus vaste qui tient compte des divers foyers ayant jadis constitué le Canada français. Le cadre spatio-temporel permet surtout d'analyser, en 
amont et en aval ce que certains ont qualifiés d' "éclatement » du Canada français, les éléments clés du discours d'enracinement afin d'y voir les points de divergence et de convergence entre nation et région dès l'émergence du projet national et la naissance d'une conscience historique dans ces espaces.

Le commentaire souligne, avec raison, la richesse et les particularités contexte pan-continental du début du $\mathrm{XX}^{\mathrm{e}}$ siècle. En effet, les raisons qui motivent les auteurs des deux sociétés historiques à rédiger en anglais se situent dans l'arène politique et sociale. Les événements qui ont lieu au cours de cette période, de même que le climat politique et les lois régissant l'éducation de langue française, influencent certainement l'élite intellectuelle locale qui rédige des textes ou fondent des sociétés d'histoire afin de faire valoir la place qu'occupent les populations de langue française dans les espaces périphériques où les francocatholiques sont minoritaires. Il importe aussi de noter que les arguments en faveur du Règlement XVII en Ontario, règlement qui cherche à éliminer l'enseignement en français dans la province, mise sur les différences entre les franco-catholiques et les autres. Une citation qui m'a toujours marqué, tirée du quotidien torontois The Mail en 1886 est très parlante à cet égard. On y qualifie les écoles de langue française de la région de Prescott et Russel dans l'Est ontarien comme « the nursuries not merely of alien tongue but of alien customs, of alien sentiments and (...) wholly of alien people ${ }^{1}$.» Que les auteurs des sociétés historiques à l'étude cherchent à présenter la "normalité » de la présence française s'inscrit parfaitement dans ce contexte, beaucoup plus large, de la «menace » catholique et française. Qui plus est, la vague de migration qui remonte au milieu du $\mathrm{XIX}^{\mathrm{e}}$ siècle et qui culmine dans les deux premières décennies $\mathrm{du} \mathrm{XX}^{\mathrm{e}}$ siècle sème sur son passage des régions entières où le français est la langue d'usage de la population qui en est à la deuxième, voire la troisième génération.

En ce qui a trait à la présence ou à l'absence d'autres groupes, il importe de noter que le cadre de ma recherche est intimement lié au territoire historique qui se dessine à partir des mouvements de migrations de la dernière moitié du 19e siècle. Ainsi, je mise davantage sur les nouveaux immigrants et sur les textes rédigés par et pour eux puisque je m'intéresse à l'émergence d'une conscience historique sur les espaces 
nouvellement colonisés. Ma réflexion suit donc les Canadiens français sur les territoires où ils s'essaiment. Il s'agit d'une posture qui se justifie en raison de la complexité évoquée cihaut dans l'analyse et l'étude de tout ce qui touche la (les) francophonie (s) et l'identité, l'appartenance, le discours. Cette prémisse explique que je ne me penche pas sur les francophones de la Louisiane ou du Missouri, qui, comme le souligne l'auteure dans son commentaire, participent à un tout autre mouvement migratoire.

En ce qui a trait au métissage, les pistes de réflexion proposées par Nicole St-Onge sont fascinantes et très inspirantes. Si elles ne cadrent pas avec les objectifs de ma recherche en cours, elles s'inscrivent néanmoins dans mon champ d'intérêt. Je souhaite d'ailleurs étudier d'autres groupes d'appartenance et leur rapport au territoire historique dans le cadre d'études postdoctorales, en commençant par les Acadiens. J'explore aussi le rapport à l'autre - Métis et Autochtone - dans l'Ouest canadien à partir des textes des premiers historiens dans le cadre de mon projet en cours qui seront explorées plus largement dans les années à venir.

\section{NOTE}

1. Cité dans Marilyn Barber et Paul-François Sylvestre, «Ontario Schools Question", Encyclopédie canadienne en ligne, page consultée le 7 juin 2017.

\section{BIBLIOGRAPHIE}

BARBER, Marilyn et Paul-François SYLVESTRE, «Ontario Schools Question », Encyclopédie canadienne en ligne, page consultée le 7 juin 2017.

FRENETTE, Yves (1998), Brève histoire des Canadiens français, Montréal, Boréal, $210 \mathrm{p}$.

PAINCHAUD, Robert (1986), Un rêve français dans le peuplement de la Prairie, Saint-Boniface, Édition des Plaines, 303 p.

ROBY, Yves (2000) Les Franco-Américains de la Nouvelle-Angleterre : Rêves et réalités, Montréal, Septentrion. 526 p.

WEIL, François (1990), Les Franco-Américains : 1860-1980, Paris, Belin, $254 \mathrm{p}$. 